\title{
Charged Particle Detection Performance Of Gas Electron Multiplier (GEM) Detectors For The Upgrade Of CMS Endcap Muon System At The CERN LHC
}

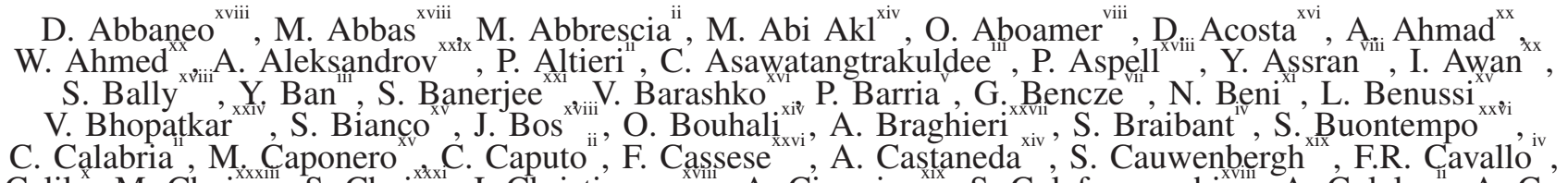

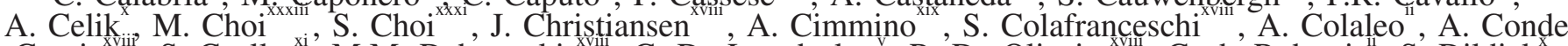

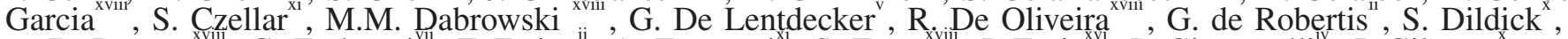

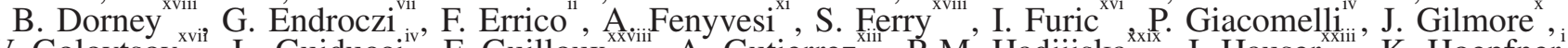

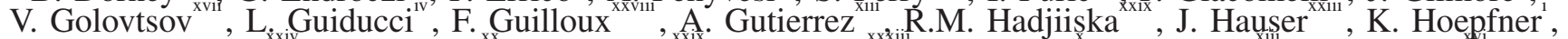

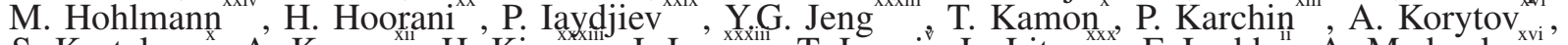

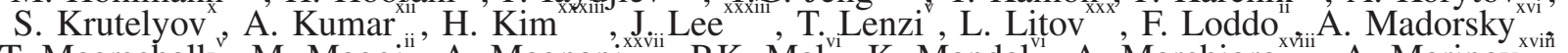

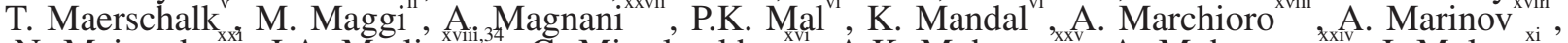

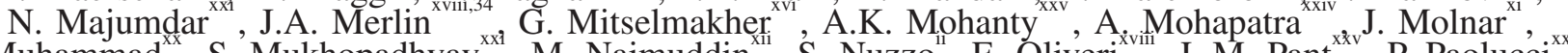

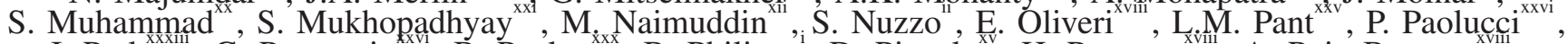

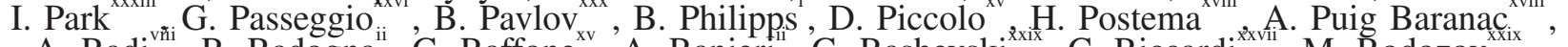

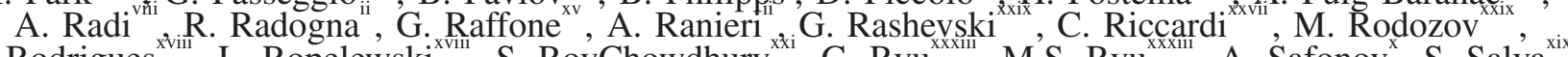

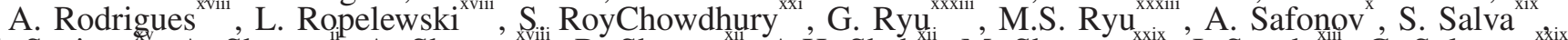

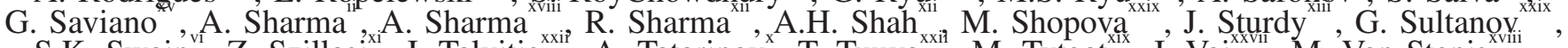

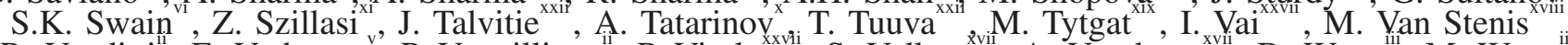

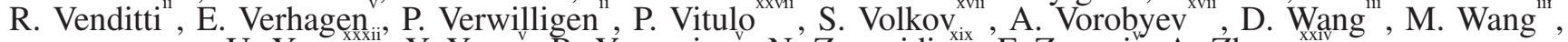
U. Yang ${ }^{\text {xii }}$, Y. Yang , R. Yonamine , N. Zaganidis ${ }^{\text {xix }}$, F. Zenoni, A. Żhang

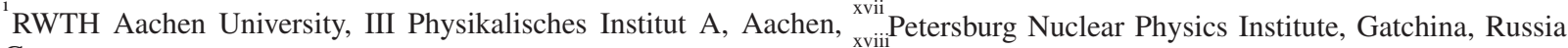
Germany

INFN Bari and University of Bari, Bari, Italy

${ }_{i 11}^{\text {ill }}$ Peking University, Beijing, China

${ }_{v}$ INFN Bologna and University of Bologna, Bologna, Italy

Universite Libre de Bruxelles, Brussels, Belgium

National Institute of Science Education and Research,

Bhubaneswar

Institute for Particle and Nuclear Physics, Wigner Research Centre for Physics, Hungarian Academy of Sciences, Budapest, Hungary

Academy of Scientific Research and Technology - Egyptian Network of High Energy Physics, ASRT-ENHEP, Cairo, Egypt

${ }_{\mathrm{x}}^{\mathrm{N}}$ Helwan University \& CTP, Cairo, Egypt

${ }_{\mathrm{xi}}$ Texas A\&M University, College Station, U.S.A.

Institute for Nuclear Research of the Hungarian Academy of garia

Sciences (ATOMKI), Debrecen, Hungary

xil University of Delhi, Delhi, India

xiii Wayne State University, Detroit, U.S.A

${ }^{\text {xiv }}$ Texas A\&M University at Qatar, Doha, Qatar

${ }^{\mathrm{xv}}$ Laboratori Nazionali di Frascati - INFN, Frascati, Italy

University of Florida, Gainesville, U.S.A. xvii Petersburg Nuclear Physics Institute, Gatchina, Russia
xviii CERN, Geneva, Switzerland
xix Ghent University, Dept. of Physics and Astronomy, Ghent, Bel-
gium

National Center for Physics, Quaid-i-Azam University Campus, Islamabad, Pakistan

xxii Saha Institute of Nuclear Physics, Kolkata, India

xxiil Lappeenranta University of Technology, Lappeenranta, Finland University of California, Los Angeles, U.S.A.

${ }_{x v}$ Florida Institute of Technology, Melbourne, U.S.A.

${ }^{x x v}$ Bhabha Atomic Research Centre, Mumbai, India

INFN Napoli, Napoli, Italy

xxviii INFN Pavia and University of Pavia, Pavia, Italy

xxix IRFU CEA-Saclay, Saclay, France

${ }^{x x i x}$ Institute for Nuclear Research and Nuclear Energy, Sofia, Bul-

${ }_{\mathrm{xxx}}$ Sofia University, Sofia, Bulgaria

${ }_{\text {xxxii }}^{\mathrm{xxx}}$ Korea University, Seoul, Korea

${ }_{\text {xxxiii }}^{\text {xxil }}$ Seoul National University, Seoul, Korea

${ }_{\text {xxxiiv }}^{\text {xxxiiv }}$ University of Seoul, Seoul, Korea

${ }^{\text {xxxiv }}$ Institut Pluridisciplinaire - Hubert Curien (IPHC), Strasbourg, France
} 


\begin{abstract}
The Compact Muon Solenoid (CMS) detector is one of the two general-purpose detectors at the CERN LHC. LHC will provide exceptional high instantaneous and integrated luminosity after second long shutdown. The forward region $|\eta| \geq 1.5$ of CMS detector will face extremely high particle rates in tens of $\mathrm{kHz} / \mathrm{cm}^{2}$ and hence it will affect the momentum resolution, efficiency and longevity of the muon detectors. Here, $\eta$ is pseudorapidity defined as $\eta=-\ln (\tan (\theta / 2))$, where $\theta$ is the polar angle measured from z-axis. To overcome these issues the CMSGEM collaboration has proposed to install new large size rate capable Triple Gas Electron Multiplier (GEM) detectors in the forward region of CMS muon system. The first set of Triple GEM detectors will be installed in the GE1/1 region $(1.6<|\eta|<2.2)$ of the muon endcap during the long shutdown 2 (LS2) of the LHC. Towards this goal, full size CMS Triple GEM detectors have been fabricated and tested at the CERN SPS, $\mathrm{H} 2$ and $\mathrm{H} 4$ test beam facility. The GEM detectors were operated with two gas mixtures: $\mathrm{Ar} / \mathrm{CO}_{2}$ (70/30) and $\mathrm{Ar} / \mathrm{CO}_{2} / \mathrm{CF}_{4}$ (45/15/40). In 2014, good quality data was collected during test beam campaigns. In this paper, the performance of the detectors is summarized based on their tracking efficiency and time resolution.
\end{abstract}

\section{INTRODUCTION}

$\mathbf{C}$ MS [1] is one of the two general-purpose detector at the CERN, LHC. As the name of detector suggest its Muon identification and reconstruction is important. Currently muon system using three detection technologies: drift tubes, Cathode Strip Chambers (CSCs) and Resistive Plate Chambers (RPCs). The endcap region rely on CSC and RPC for $|\eta|<1.6$. For higher $\eta>1.6$, the muon system has limited redundancy and only CSCs are installed. After LS2, when we run at higher luminosity, the particle rate in the forward region is expected to reach several tens of $\mathrm{kHz} / \mathrm{cm}^{2}$ and the integrated charge will reach several $\mathrm{C} / \mathrm{cm}^{2}$. So, After LS2, it is not possible to use the RPC in region $|\eta|>1.6$. To overcome this limitations, the CMS-GEM collaboration proposed the Gas Electron Multiplier (GEM)[3] as one of most promising candidate to upgrade the high- $\eta$ region of the forward muon system. GEM based detectors have demonstrated excellent spatial $(100 \mu m)$ and timing resolution $(\sim 5 n s)$, and are able to cope with particle rate up to $10 \mathrm{MHz} / \mathrm{cm}^{2}$, which make them a promising candidate to complement CSC chambers in high $-\eta$ region. Therefore, their use in CMS experiment will improve muon momentum resolution for high $p_{T}$ muon and to provide overall highly efficient muon and trigger tracking capabilities in this region.

\section{Detector Description}

The GEM prototype for CMS detector is known as GE1/1. It is trapezoidal in shape with active area of $990 \times(220-$ 445) $\mathrm{mm}^{2}$. This size is imposed by the geometry of the vacant high- $\eta$ area in CMS muon endcap. GE1/1 chamber hosts a Triple-GEM detector with a $3 / 1 / 2 / 1 \mathrm{~mm}$ (drift/transfer $1 /$ transfer $2 /$ induction) electrode gap configuration, as shown in Fig. 1. The GEM foil $(50 \mu m$ thick kapton foil with $5 \mu m$ copper on both sides) consists of a thin kapton foil, metal-clad on both side, with a high density of chemically pierced holes. By applying suitable potential difference between two sides, this mesh can act as an amplifier for electrons released by ionization of the gas. The detector readout board is divided into

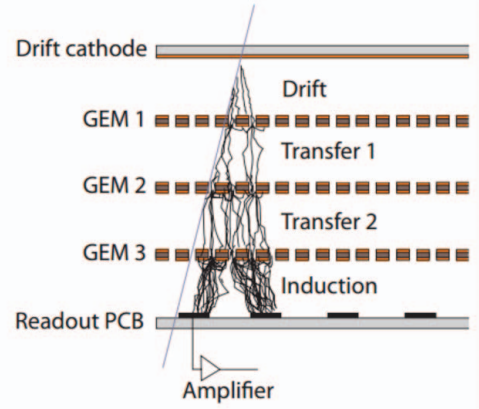

Fig. 1. Generic triple-GEM chamber, showing drift, transfer, and signal induction gap regions within the detector.

eight $\eta$-partitions with 384 strips each oriented radially along the long side of the detector with a pitch varying from $0.6 \mathrm{~mm}$ (short side) to $1.2 \mathrm{~mm}$ (long side). Each partition is subdivided along the $\phi$-coordinate into three readout sectors with 128 strips or channels each. During test beam we scanned three different sectors of GE1/1, i.e. $(i \eta, i \phi)=\{(1,2),(5,2),(8,2)\}$. In Fig. 2 red and yellow color shows which sector of GE1/1's are exposed to the beam. Red sectors are taken with gas $\mathrm{Ar} / \mathrm{CO}_{2} / \mathrm{CF}_{4}(45 / 15 / 40)$ while yellow sector is taken with gas $\mathrm{Ar} / \mathrm{CO}_{2}(70 / 30)$.

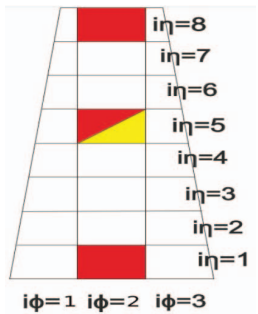

Fig. 2. Different $(i \eta, i \phi)$ sectors of full size GE1/1 detector prototype.

\section{TEST BEAm SET-UP}

$\mathbf{T}$ HE GE1/1 detector were tested using $\sim 150 \mathrm{GeV}$ Muon beam at the CERN SPS test beam facility during OctoberDecember 2014. The test beam set-up is shown in Fig. 3. The set-up consists of three scintillators, three trackers and

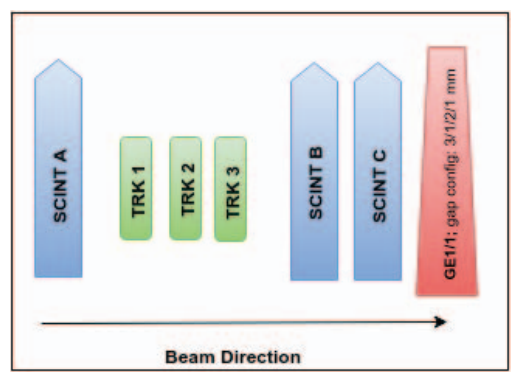

Fig. 3. Schematic of the test beam set-up.

a GE1/1 prototype. The trackers are triple-GEM detectors with a $10 \times 10 \mathrm{~cm}^{2}$ active area. Each tracker has 256 strips in both horizontal (y-coordinate) and vertical (x-coordinate) directions transverse to the beam. Trackers acts as muon 
telescope which helps us to reconstruct the tracks and reduce backgrounds. The trigger comes from the coincidence of three photo-multiplier tubes with mounted scintillators. The GE1/1 prototype is installed on a movable table for scanning different sectors. Fig. 4 shows a beam profile of the muon beam as reconstructed with three trackers. And Fig. 5 represents the
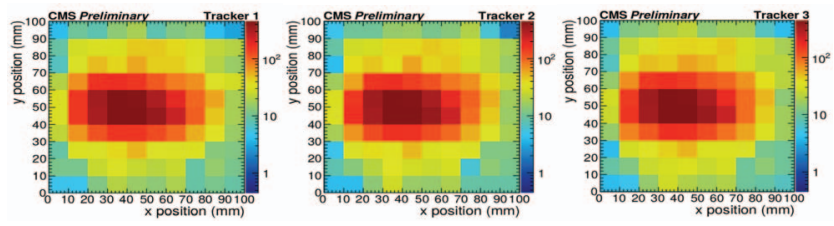

Fig. 4. Profile plot for first, second and third tracker. This is plotted from one of run taken during test-beam.

tracker and GE1/1 hit positions along $\mathrm{x}$ and $\mathrm{y}$ direction.
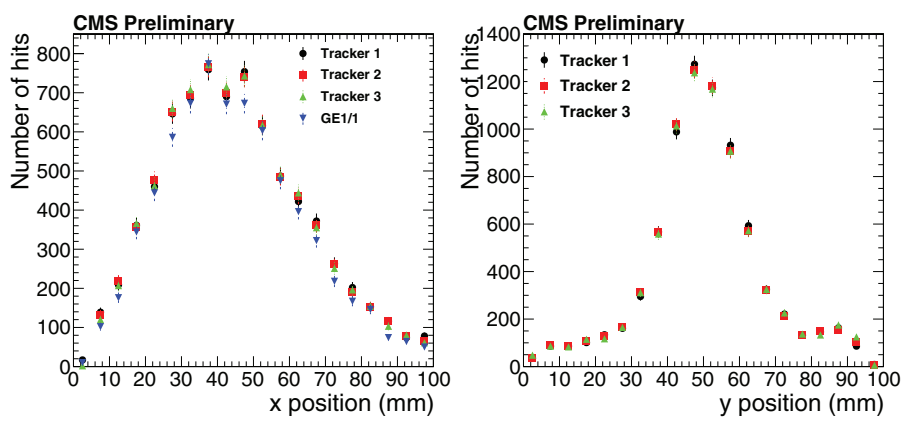

Fig. 5. Tracker hit distribution along $x$ and $y$ axis and GE1/1 hit distribution along $\mathrm{y}$. This is plotted from one of run taken during test-beam.

\section{EFFICIENCY MEASUREMENT}

$\mathbf{E}$ FFICIENCY, $\epsilon$ is one of the most important parameter for the gaseous detectors. Here it is defined as

$$
\epsilon=\frac{N_{G E 1 / 1+T r k}}{N_{T r k}}
$$

where $N_{\text {Trk }}$ is the number of reconstructed events by using a linear fit $y=m x+b$ fit to the tracker hit positions, in the tracker with normalized $\chi^{2}<10 . N_{G E 1 / 1+T r k}$ is the number of reconstructed events for which an actual hit is found in the GE1/1 within $5 \mathrm{~mm}$ of extra-plotted track. We are showing here the efficiency as a function of $E_{\text {gain }}$, Fig. 6. Where $E_{\text {gain }}$ is defined as

$$
E_{\text {gain }}=\frac{I \times R_{\text {avg }}^{\text {gap }}}{D}
$$

Where $I$ is current supplied to the HV divider, $R_{\text {avg }}^{\text {gap }}$ is the average gap resistance of GE1/1, and D is the thickness of GEM foil. Fig. 6 is showing the efficiency w.r.t. two different gas mixtures $\mathrm{Ar} / \mathrm{CO}_{2}(70 / 30)$ at sector $(i \eta, i \phi)=(5,2)$ and $\mathrm{Ar} / \mathrm{CO}_{2} / \mathrm{CF}_{4}(45 / 15 / 40)$ scanned at three different sectors $(i \eta, i \phi)=\{(1,2),(5,2),(8,2)\}$. We achieved very good efficiency of $\sim 98 \%$ in all cases. While for gas mixture $\mathrm{Ar} / \mathrm{CO}_{2}$ the threshold is shifted as compared to the $\mathrm{Ar} / \mathrm{CO}_{2} / \mathrm{CF}_{4}$ because at fixed high voltage operating point, the effective gain with $\mathrm{Ar} / \mathrm{CO}_{2}$ mixture is approximately one order of magnitude higher than $\mathrm{Ar} / \mathrm{CO}_{2} / \mathrm{CF}_{4}$ mixture.

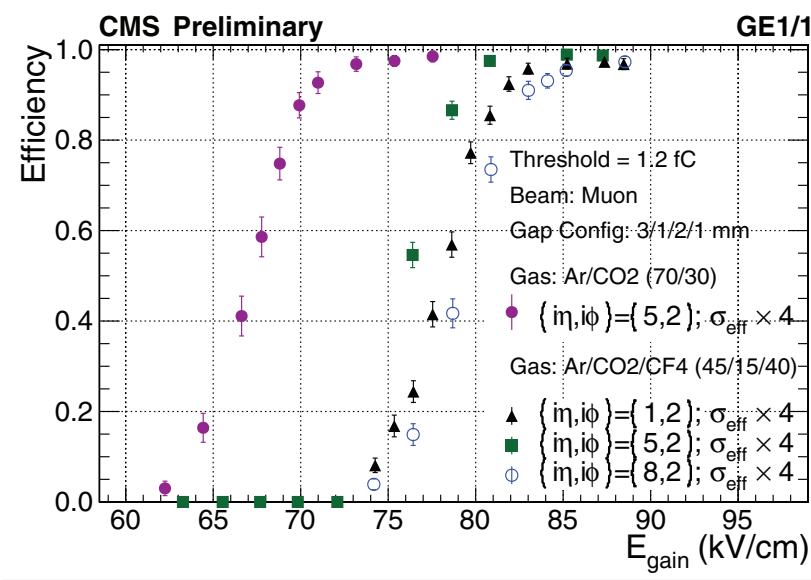

Fig. 6. Efficiency w.r.t. $E_{\text {gain }}$ for two different gases and three different $(i \eta, i \phi)$ sectors.

\section{Time Resolution}

$\mathbf{T}$ IME resolution of a detector is defined as the minimum gate width necessary on the detection electronics for full efficiency. Experimentally, the time resolution is the rms of the Gaussian distribution of the time taken by particle to reach detector from scintillator. Along with the fast $40 \mathrm{MHz}$ (25ns) clock pulse have been used to cope with the LHC bunch crossing. So, the detector time response is modelled as the Gaussian function, $f(t)$, convoluted with a square wave, $g(t)$, having pulse length $f_{c l k}=25 n s$ to represent discrete sampling. The convolution of the two function is

$$
(f * g)(t)=A \cdot \sigma \sqrt{\frac{\pi}{2}}\left(\operatorname{erf}\left(\frac{u_{+}}{\sigma \sqrt{2}}\right)-\operatorname{erf}\left(\frac{u_{-}}{\sigma \sqrt{2}}\right)\right)
$$

where $A$ is the amplitude of the Gaussian function, $\sigma$ is the standard deviation of Gaussian, and $u_{ \pm}=t-t_{0} \pm \frac{f_{c l k}}{2}$. We fitted the experimental data with this convoluted function. From, the fit we extract the time resolution of the detector before the convolution. The time resolution as a function of $E_{\text {drift }}$ is shown in Fig. 7. The time resolution with $\mathrm{Ar} / \mathrm{CO}_{2}$ (70/30) is higher for lower values of $E_{\text {drift }}$. However for any given point on the $\mathrm{Ar} / \mathrm{CO}_{2}$ curve has a gain approximately one order of magnitude higher gain than the corresponding gain with $\mathrm{Ar} / \mathrm{CO}_{2} / \mathrm{CF}_{4}(45 / 15 / 40)$. We are able to reach faster timing at lower gains with the addition of the $C F_{4}$ and this is important from the point of view of detector safety because this will allow us to operate the detector at lower gains, hence reducing the discharge probability.

\section{CONCLUSION AND SUMMARY}

A $\mathrm{T}$ the test beam facility at CERN, we studied the efficiency and time response of the GE1/1 detector with the exposure of muon beam. We were able to get very good efficiency $\sim 98 \%$ and time resolution $\sim 7 \mathrm{~ns}$ in case of both gas mixtures $\mathrm{Ar} / \mathrm{CO}_{2}(70 / 30)$ and $\mathrm{Ar} / \mathrm{CO}_{2} / \mathrm{CF}_{4}$ $(45 / 15 / 40)$. In this test beam campaign it was shown that we can operate GEM detectors without using $C F_{4}$, while keeping same efficiency and timing resolution as with gas mixture $\mathrm{Ar} / \mathrm{CO}_{2} / \mathrm{CF}_{4}$. 


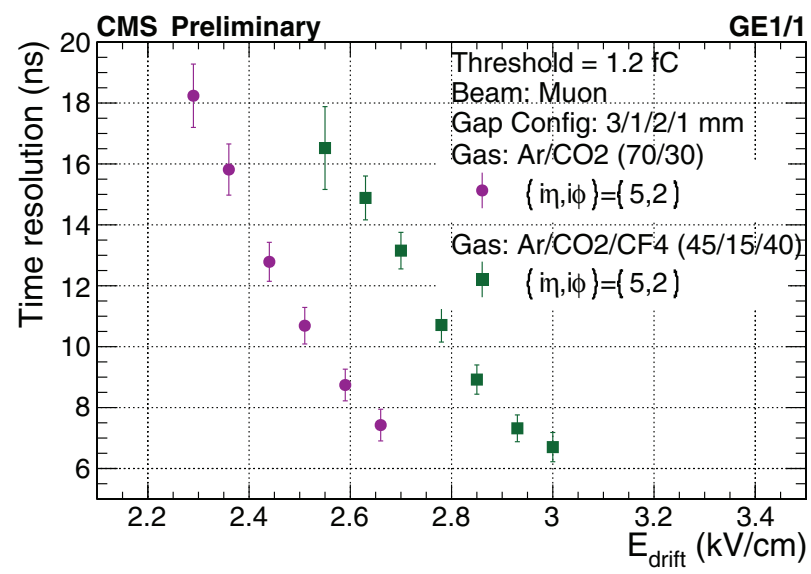

Fig. 7. Time-resolution w.r.t. $E_{\text {drift }}$ for two different gases.

\section{ACKNOWLEDGMENT}

We gratefully acknowledge support from FRS-FNRS (Belgium), FWO-Flanders (Belgium), BSF-MES (Bulgaria), BMBF (Germany), DAE (India), DST (India), INFN (Italy), NRF (Korea), QNRF (Qatar), and DOE (USA).

\section{REFERENCES}

[1] S. Chatrchyan et al. [CMS Collaboration], "The CMS experiment at the CERN LHC," JINST 3 (2008) S08004. doi:10.1088/17480221/3/08/S08004

[2] H. W. Wulsin, "Performance of the Cathode Strip Chamber endcap muon detectors in Run 2," arXiv:1510.08908 [physics.ins-det].

[3] F. Sauli, "GEM: A new concept for electron amplification in gas detectors", Nucl. Instrum. Meth. A 386 531, 1997.

[4] CMS GEM Collaboration, CMS Technical Design Report for the muon endcap GEM upgrade, CMS-TDR-15-001-001. 